\title{
Tensiones entre el saber pedagógico en evaluación y la práctica docente en escuelas vulnerables de la Comuna de Arica
}

\author{
Tensions Between Pedagogical Knowledge in Evaluation and Teaching Practice in \\ Vulnerable Schools of the Arica Commune
}

\section{Tensões entre o saber pedagógico em avaliação e prática docente em escolas vulneráveis da Comuna de Arica}

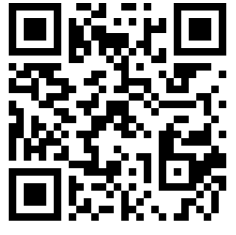

Katherine Roberts-Sánchez Universidad Católica de la Santísima Concepción Concepción, Chile kroberts@ucsc.cl

(iD) https://orcid.org/0000-0003-4353-1082

David Rodríguez-Gómez Universidad Autónoma de Barcelona

Barcelona, España david.rodriguez.gomez@uab.cat https://orcid.org/0000-0001-9845-0744

Patricia Silva Universidad de Lleida

Barcelona, España patricia.silva@udl.cat iD https://orcid.org/0000-0001-5651-296X

Recibido • Received • Recebido: 11 / 12 / 2018

Corregido • Revised • Revisado: 27 / 05 / 2020

Aceptado • Accepted • Aprovado: 03 / 08 / 2020

Resumen: Este artículo analiza las prácticas evaluativas del profesorado que se desempeña en quince escuelas vulnerables de la comuna de Arica, ubicada en el extremo norte de Chile. Se trata de un estudio cualitativo cuyos datos han sido recogidos mediante entrevistas semiestructuradas y grupos de discusión. Para procesar la información, se realizó análisis de contenido siguiendo las directrices de la teoría fundamentada. Los resultados muestran que, si bien, a lo largo de su trayectoria profesional, el profesorado ha ido transitando desde una práctica evaluativa tradicional, a una cada vez más auténtica, aún existen tensiones entre su discurso sobre la evaluación y sus prácticas evaluativas. La evidencia de esas tensiones recomienda conectar la experiencia educativa y evaluativa del aula con asuntos relevantes de la vida del estudiantado. Se trata de promover entre el profesorado prácticas evaluativas sensibles a los contextos escolares, que incorporen recursos afectivos en su implementación.

Palabras claves: Evaluación del estudiantado; escuelas vulnerables; formación del profesorado; prácticas docentes; saber pedagógico; recursos afectivos. 
http://doi.org/10.15359/ree.24-3.15

http://www.una.ac.cr/educare

educare@una.ac.cr

\begin{abstract}
This article analyzes the evaluative practices of teachers working in fifteen vulnerable schools in the Arica Commune, located in the extreme north of Chile. This is a qualitative study whose data has been collected through semi-structured interviews and discussion groups. To process the collected information, content analysis was performed following the guidelines of the Grounded Theory. The results show that, although teachers have been transitioning from a traditional evaluative practice to an increasingly authentic one throughout their professional career, there are still tensions between their discourse on evaluation and their evaluative practices. The evidence of these tensions recommends connecting the educational and evaluative experience of the classroom with relevant issues in the student's life. The aim is to promote evaluative practices sensitive to school contexts among teachers, incorporating affective resources in their implementation.
\end{abstract}

Keywords: Student evaluation; vulnerable schools; teacher training; teaching practices; pedagogical knowledge; affective resources.

Resumo: Este artigo analisa as práticas avaliativas dos professores que trabalham em quinze escolas vulneráveis na comuna de Arica, localizada no extremo norte do Chile. Este é um estudo qualitativo cujos dados foram coletados por meio de entrevistas semiestruturadas e grupos de discussão. Para processar as informações, a análise de conteúdo foi realizada seguindo as diretrizes da teoría fundamentada. Os resultados mostram que, embora, ao longo de sua carreira profissional, o grupo de professores tenham transitado de uma prática avaliativa tradicional para uma prática cada vez mais autêntica, ainda existem tensões entre seu discurso sobre avaliação e suas práticas avaliativas. A evidência dessas tensões recomenda conectar a experiência educacional e avaliativa da sala de aula com questões relevantes na vida dos e das estudantes. O objetivo é promover práticas avaliativas sensíveis aos contextos escolares entre os professores, incorporando recursos afetivos em sua implementação.

Palavras-chave: Avaliação dos estudantes; escolas vulneráveis; formação de professores; práticas de ensino; saber pedagógico; recursos afetivos.

\title{
Introducción
}

La historia de la evaluación, en el ámbito de la educación escolar, evidencia cambios de manera sostenida y continua a través del tiempo (Perassi, 2014). Desde la concepción más tradicional, investigar sobre las prácticas evaluativas es adentrarse en uno de los ámbitos más complejos de la acción pedagógica, tanto para estudiantes como para el propio profesorado. Para cada estudiante es complejo porque suele exponerle al juicio docente frente a sus pares, ya que genera un espacio en el que se legitima a "otro ser" para que juzgue su desempeño, sin mediar acompañamiento en la superación o ajuste de ese desempeño. En el caso del profesorado, este aspecto también suele ser complejo, porque el diseño y aplicación de los instrumentos de evaluación toma mucho tiempo y el sistema administrativo escolar exige un número definido de calificaciones, en un período de tiempo acotado. 
http://doi.org/10.15359/ree.24-3.15

Actualmente, la evaluación auténtica se basa en un concepto más amplio de lo que significa evaluar y se representa como un proceso y no como una foto del momento. Esta nueva concepción evaluativa releva la importancia de evaluar al estudiantado, al valorar su aprendizaje en una situación o contexto de la vida real y en condiciones que le permitan mostrar la riqueza del aprendizaje tanto a nivel personal como también social (Vallejo Ruiz y Molina Saorín, 2014). Es decir, desafía al profesorado a evaluar mediante situaciones reales de aprendizaje, ya que solo las valoraciones contextualizadas en la realidad escolar podrán entregar los elementos apropiados y significativos para la reflexión y aprendizaje de los grupos de estudiantes (Quesada Serra et al., 2017).

Tanto las prácticas tradicionales (Hernández-Nodarse, 2017) como las auténticas son producto del saber pedagógico que surge de interacciones entre las disposiciones personales internas y el contexto sociocultural donde se desempeña el profesorado (Sotos y López, 2015). El saber pedagógico en evaluación es construido durante la formación inicial docente y las experiencias pedagógicas previas al ejercicio formal de la profesión. También considera la práctica profesional docente y se operacionaliza en maneras de evaluar que, habitualmente, denotan tensión entre lo que expresa por el profesorado y lo que efectivamente realiza en el aula (Reyes et al., 2013).

Este saber pedagógico también ha sido erigido por el profesorado, al considerarse la diversidad de prácticas evaluativas implementadas, fruto de las particularidades y características que tienen y demandan las poblaciones estudiantiles de diferentes contextos escolares, por ejemplo, de lo que conocemos como escuelas vulnerables. En Chile, las escuelas vulnerables son establecimientos educacionales públicos, financiados por el Estado, que reciben estudiantes en condición de extrema pobreza y que son categorizados anualmente por el Ministerio de Educación, según el bajo nivel socioeconómico de las familias, la formación educativa de los padres y madres, y las condiciones de riesgo que afectan al estudiantado y que, eventualmente, lo expone a la deserción del sistema escolar o al fracaso académico (Díaz López y Pinto Loría, 2017; Forján y Morelatto, 2018; Infante et al., 2011).

El sistema educativo chileno ha incrementado su población estudiantil de manera explosiva en los últimos años (Santelices et al., 2015). Este aumento repentino del número de estudiantes ha ido acompañado de un creciente interés por avanzar en el fortalecimiento de la calidad de la educación. No obstante, a pesar de la importancia de la acción docente en la mejora de la calidad educativa (Torres, 2018), son escasas las propuestas que, desde un ámbito teórico o práctica, se han preocupado por profundizar en el análisis del saber pedagógico evaluativo de docentes que se desempeñan en escuelas vulnerables.

Seguramente esto acontece porque, como señala Suárez (2017), sus prácticas “...son descalificadas como parte del conjunto de anécdotas triviales que los docentes usan para contarse entre sí lo que les pasa en las aulas y escuelas" (p. 45). 
http://doi.org/10.15359/ree.24-3.15

http://www.una.ac.cr/educare

educare@una.ac.cr

Ante este escenario y como resultado de las reflexiones que se han realizado sobre la importancia de la evaluación, la concepción pedagógica que promueve una evaluación auténtica y del saber pedagógico construido en contextos escolares vulnerables, el estudio que se presenta apunta a responder las siguientes interrogantes: ¿cómo ha construido el profesorado en estudio su saber pedagógico evaluativo?, ¿cómo han ido modificando sus prácticas evaluativas a través del ejercicio profesional en contextos escolares vulnerables?

Concretamente, el propósito central de esta investigación es analizar el saber pedagógico en evaluación del profesorado que se desempeña en escuelas vulnerables, identificando las maneras en que el saber pedagógico orienta sus prácticas evaluativas y evidenciando los requerimientos a la formación inicial docente que demanda el profesorado que se desempeña en este contexto.

\section{Construcción del saber pedagógico evaluativo}

Existen diferentes saberes, de carácter externo o interno que, en conjunto, orientan la acción docente y dan cuerpo al saber pedagógico: saber profesional (saberes transmitidos en la formación inicial docente); saber disciplinario (que refieren a los diversos ámbitos del conocimiento); saber curricular (que se expone en forma de programas escolares y que el profesorado debe aplicar) y, por último, saber práctico o experiencial, que en palabras de Tardif (2004) se define como "núcleo a partir del cual los profesores tratan de transformar sus relaciones de carácter exterior con los saberes en relaciones de carácter interior con su propia práctica" (p. 41).

De acuerdo con Solary Díaz (2018), el saber pedagógico es un saber inherente al profesorado, que se construye "producto de la biografía y experiencia del docente, de sus conocimientos actuales y de su relación activa con la práctica" (p. 2). Se erige en el transcurso de la vida como estudiante, en las prácticas pedagógicas contempladas en la malla curricular de la formación inicial, en el mundo laboral y en otras situaciones cotidianas; en las experiencias de cada persona y en sus relaciones sociales. Todo de acuerdo al contexto sociocultural donde está inmerso profesionalmente (Sotos y López, 2015). Es un saber teórico que origina el mismo educador a partir de sus propios procesos de reflexión docente, contextualizado a una comunidad escolar determinada, y se plasma en la práctica evaluativa (López-Lozano et al., 2017).

Ibáñez Salgado (2014) va más allá al plantear que existe una especie de memoria del saber pedagógico en los registros que realiza el profesorado durante su trabajo cotidiano de aula. Según Cárdenas Pérez et al. (2012), estos registros comprenderían percepciones acerca de su trabajo docente, producto del "proceso de aprendizaje y enseñanza, anécdotas, experiencias, aciertos y desaciertos de los procesos formativos" (p. 483) y también de algunas visiones sobre el comportamiento y desempeño estudiantil. 
http://doi.org/10.15359/ree.24-3.15

\section{Evaluación auténtica}

La evaluación escolar se ha ido modificando en las últimas décadas, avanzando desde una mirada tradicional a la actual concepción: evaluación auténtica. En ese sentido, se ha transitado desde la evaluación como acción enjuiciadora a un proceso que se nutre del contexto, de los propios sujetos (evaluados y evaluadores) y de sus acciones e implicancias, para constituirse en posibles agentes transformadores de la sociedad desde el espacio educativo (Murillo e Hidalgo, 2016).

Vallejo Ruiz y Molina Saorín (2014) señalan que será auténtica en la medida que relacione la experiencia educativa con temas importantes de la vida en diferentes ámbitos e integre, en su aplicación, la coevaluación y autoevaluación. Complementariamente, Brown (2015) señala que "se basa en la especificación de unos resultados de aprendizaje significativos y concretos según el nivel de cualificación perseguido, que después se reflejan completamente en las tareas que los estudiantes deben llevar a cabo para demostrar su competencia" (p. 3).

La evaluación auténtica, entendida como actividad evaluativa derivada de una situación de la vida real (Ravela, 2015), se distingue no solo por incluir como contexto evaluativo estos escenarios, sino que también por incorporar problemas significativos que no se solucionan con respuestas sencillas seleccionadas de un repositorio de preguntas. Requiere de un ajuste o modificación de las prácticas evaluativas del profesorado, lo que implica tener un conocimiento actualizado del sentido que posee la evaluación y disposición a querer cambiar estas prácticas (Moreno Olivos, 2017). La evaluación auténtica demanda que el profesorado pueda motivar a los estudiantes a aprender y aplicar lo aprendido en situaciones y contextos reales, asignándoles tareas significativas y relevantes, las cuales se pueden convertir en una desafiante oportunidad de aprendizaje (Brown, 2015).

Esta idea genera que el profesorado pueda tener presente que lo más importante no es evaluar, ni hacerlo con la frecuencia requerida, sino saber cuáles son los propósitos del proceso de aprendizaje-enseñanza, entender las expectativas existentes en función del nivel de logro esperado (Vallejo Ruiz y Molina Saorín, 2014) y, en qué momento de la clase, curso o etapa, es más propicio evaluar.

\section{Metodología}

La investigación que se presenta se construyó desde la metodología cualitativa de tipo descriptivo. Tuvo, como propósito, adentrarse en el mundo personal de los sujetos en estudio y perseguir consenso en los distintos significados, utilizando como criterio el acuerdo intersubjetivo en el contexto educativo (Sabino, 2014). El método de investigación propuesto para desarrollar esta tarea, entendido por Herrera (2017) como la forma característica de investigar determinada por la intención y enfoque que la orienta, fue la teoría fundamentada (TF). En la TF, los datos 
http://doi.org/10.15359/ree.24-3.15

http://www.una.ac.cr/educare

educare@una.ac.cr

constituyen categorías preliminares, luego códigos conceptuales y, posteriormente, conceptos centrales o teoría sustantiva y se orienta a descubrir una realidad a partir del "significado" que tiene para las personas desde su propio contexto, con lo cual genera conocimientos y aumenta la comprensión de ese entorno (Carrero et al., 2012). Además, la recogida y análisis de la información se desarrolla de forma paralela y constante (Strauss y Corbin, 2002).

La muestra inicial estuvo constituida por veintidós docentes, hombres y mujeres, pertenecientes a quince establecimientos educacionales, de dependencia municipal, ubicados en la comuna de Arica. El tamaño de la muestra no fue definido a priori, sino que se fue ajustando durante la recogida de información en coherencia con el criterio de saturación teórica definido por la TF. La saturación se evidenció en el momento en que los relatos aportados por el profesorado y el análisis ulterior de las categorías no evidenciaban propiedades nuevas, constituyéndose en categorías centrales de análisis o códigos teóricos (Monge Acuña, 2015).

Con posterioridad a la aplicación y análisis de las entrevistas, se realizó un grupo de discusión en el que participaron ocho docentes que se seleccionaron con los mismos criterios que en el primer instrumento, con el fin de triangular la información recogida y resguardar que las ideas expresadas por los sujetos consultados en la entrevista semiestructurada tuvieran una correcta interpretación.

Las personas entrevistadas fueron escogidas de manera intencionada. Los criterios de selección fueron: i) enseñar más de una materia en el ciclo básico de formación escolar ( $1^{\circ}$ a $8^{\circ}$ básico en Chile); ii) tener un mínimo de cinco años de ejercicio profesional en aula y desempeñarse laboralmente, al menos, durante dos años en la misma escuela de la comuna de Arica. Respecto de la selección de los establecimientos escolares, debían disponer de un alto índice de vulnerabilidad escolar; esto es, establecimientos educacionales que reciben alumnado en condición de extrema pobreza.

La información aportada por el profesorado fue recogida a través de entrevista semiestructurada y un grupo de discusión. El corpus estuvo compuesto por una masa textual aproximada de 45000 palabras, que fue analizada en el programa Atlas ti. La organización de la generación de datos o proceso de codificación fue realizado en tres etapas: codificación abierta, codificación axial y codificación selectiva (Strauss y Corbin, 2002).

\section{Resultados}

En acuerdo con los criterios de rigor cualitativo planteados por Noreña et al. (2012), y en coherencia con la orientación o significado que tienen y el procedimiento de acción, esta investigación asume los siguientes criterios de rigor y calidad: fiabilidad, credibilidad, transferibilidad, dependencia y confirmación. 
http://doi.org/10.15359/ree.24-3.15

En concordancia con las preguntas centrales que guiaron y articularon la investigación, los resultados obtenidos dan cuenta del camino recorrido por el profesorado en la construcción de su saber evaluativo actual y han sido agrupados en dos temáticas principales: Saber pedagógico en evaluación y lecciones aprendidas del profesorado. La primera, da cuenta del saber pedagógico evaluativo construido por docentes en un contexto escolar vulnerable, mientras que la segunda pretende informar de algunas lecciones del profesorado que, en calidad de vacíos formativos, han aprendido.

\section{a) Saber pedagógico en evaluación}

En relación con el saber evaluativo declarado por el profesorado, los datos disponibles permiten asociar dos códigos abiertos: Experiencia profesional docente en evaluación y fundamentación de la evaluación usada.

El primer código, que emana del procesamiento de los datos, es el de experiencia profesional docente en evaluación, que hace referencia al saber pedagógico construido por el profesorado desde su formación inicial hasta su vida laboral actual, lo que incluye: experiencias en los centros de educación superior en los que estudiaron, experiencias formativas prácticas como docente en formación y reflexiones como profesional en ejercicio de un establecimiento educacional vulnerable.

A partir del discurso del profesorado, se puede concluir que, en esta trayectoria vivencial y profesional, su saber pedagógico evaluativo ha ido transitando desde prácticas caracterizadas por la monotonía, en donde primaban las evaluaciones sumativas y en las cuales eran los únicos sujetos evaluadores de estudiantes, a prácticas evaluativas más auténticas donde han incorporado, por ejemplo, diálogo con sus estudiantes, evaluaciones de proceso y entre pares.

La sensación que experimentan al mirar el camino recorrido es de agrado y de conciencia de cambio de sus propias prácticas evaluativas, sienten a ratos orgullo de inducir procesos evaluativos encaminados a que sus estudiantes valoren su desempeño y el de sus compañeros y compañeras. No disimulan cómo, en este proceso, han sido capaces de implementar prácticas evaluativas que les permiten obtener información sobre el aprendizaje del proceso formativo, evaluar conductas observables, considerar las características de sus estudiantes y el contexto escolar vulnerable de su espacio laboral, lo cual les lleva a favorecer el desarrollo de habilidades para la vida de sus estudiantes. Características que son definidas a partir de frases como "... hago una evaluación conjunta, colectiva, de habilidades en proceso en el aula, no estandarizada..." (Entrevista 1) y"... me he dado cuenta de que los resultados han sido positivos en varios aspectos no solo en aspecto cognitivo sino más bien en habilidades para los niños para la vida..." (Entrevista 3). 
http://doi.org/10.15359/ree.24-3.15

http://www.una.ac.cr/educare

educare@una.ac.cr

Del relato también se desprende cómo es que el profesorado ha ido paulatinamente incorporando la autorreflexión respecto a los alcances que poseen sus prácticas evaluativas, especialmente resulta relevante la valoración que realizan de la flexibilidad y pertinencia que debe acompañar este proceso, lo que los ha obligado a ajustar los instrumentos evaluativos a los requerimientos y demandas propias del contexto escolar vulnerable de sus estudiantes, como se desprende del siguiente relato:

Reflexiono siempre entorno a mi práctica porque hoy creo que es muy importante poder hacerlo ya que me he dado cuenta lo importante que es generar instrumentos evaluativos coherentes al contexto escolar en que me desenvuelvo, porque debe haber coherencia entre ellos y este entorno vulnerable. Lo único que eso ha hecho y ha provocado en mí, es mejorar mi trabajo. (Entrevista 5)

En forma consistente con la búsqueda de sintonía con el estudiantado y su contexto escolar vulnerable, el personal docente entrevistado señala que ha implementado una serie de recursos afectivos en sus prácticas evaluativas, que les han dado muy buenos resultados. Estos recursos afectivos, más allá de utilizar un lenguaje más cercano con sus estudiantes, consisten en considerar la evaluación como una oportunidad de diálogo y una relación de ayuda entre personas que persiguen un mismo fin. La conciben como una forma más cercana de obtener información válida, para ir acompañando al alumnado en sus complejos procesos de aprendizaje, desde un ejercicio pedagógico dialogado que dista de una acción punitiva, ni menos pensando exclusivamente en una calificación.

La incorporación de recursos afectivos en sus prácticas evaluativas es concebida por el profesorado como un proceso que lo hace sentirse más humano, cercano y empático con las necesidades que poseen sus estudiantes, lo que - de acuerdo conl relato del propio profesoradoel estudiantado capitaliza en mayor confianza y, como consecuencia, tributa en más y mejores aprendizajes.

Así se desprende de los siguientes relatos: "...he aprendido a modificar mi estructura, mis evaluaciones, como te digo a ser más humana..." (Entrevista 7) y "...en los colegios vulnerables, justamente hay más de una realidad donde los alumnos requieren más atención y cariño y trato de generar eso en los niños, porque el afecto también te produce confianza y cuando hay confianza hay aprendizaje..." (Entrevista 6). Estos recursos afectivos contribuyen a concebir la evaluación como un proceso que pretende no solo una preocupación por la construcción de conocimientos por parte del estudiantado, sino que también -como veremos en el segundo código- de habilidades y actitudes, lo que resulta coherente con las exigencias curriculares que posee el marco curricular nacional de Chile. 
http://doi.org/10.15359/ree.24-3.15

El segundo código que emerge del análisis de los datos esla fundamentación de la evaluación usada. Da cuenta de los argumentos que utiliza el profesorado para justificar el proceso de transición de sus prácticas evaluativas desde las más tradicionales a las más auténticas. Entre las fundamentaciones empleadas por los sujetos entrevistados, destacan la preocupación por incorporar prácticas que ayuden a sus estudiantes a superar falencias específicas, producto de su condición de vulnerabilidad, que resultaban imposibles de evaluar desde una concepción tradicional: su timidez y su expresión oral descendida. Además, la preocupación por incorporar procedimientos que promuevan la evaluación de los procesos de construcción de conocimiento y, por cierto, la intención de valorar no solo contenidos, sino también habilidades y actitudes, pensadas no solo desde las exigencias curriculares, sino considerando las necesidades que poseen sus estudiantes y el contexto escolar vulnerable en el que se insertan.

De esta manera, el profesorado declara que ha implementado evaluaciones que promueven destrezas orales (disertaciones, representaciones, diálogos, pruebas orales), debido a que sus estudiantes tienen dificultades para expresarse oralmente, lo que ha sido corroborado en los bajos resultados de las pruebas nacionales en las que han participado. Al respecto, una profesora indica: "por la vulnerabilidad social-cultural, a ellos les costaba un poco más expresarse oralmente, les era más fácil demostrar lo que habían entendido de manera escrita, por eso les he dado la oportunidad que lo hagan de manera oral" (Entrevista 8).

También reconocen que han incorporado procedimientos evaluativos que les han permitido evaluar integralmente a sus estudiantes, poniendo acento en procesos formativos donde el intercambio de experiencias, el trabajo colaborativo, el debate y la elaboración de productos de manera cooperativa han resultado experiencias que valoran profundamente. Se han potenciado los trabajos prácticos, debido a que estas experiencias hacen que el estudiantado elabore un producto a través de una secuencia de actividades que facilita la evaluación de proceso y visibiliza lo aprendido. Una docente reconoce la consideración vinculada a las características de sus estudiantes:

En vez de evaluar a los estudiantes con una prueba escrita sumativa, los evalúo a través de una disertación, una maqueta, un trabajo que hace que aprendan mucho más que en un examen escrito. El niño aprende más haciendo que escribiendo. (Entrevista 7)

Finalmente, la evaluación de habilidades y actitudes es un ámbito también valorado por el profesorado para justificar el proceso de transición en el que se encuentra. Esto, porque les permiten incorporar recursos afectivos como el diálogo, la empatía y el buen trato a estudiantes, al incluir dimensiones formativas que no son posibles de evaluar con una concepción más tradicional. Al menos así se desprende del siguiente relato "... a veces a través de mi evaluación de los trabajos que ellos realizan en grupo, salen cosas de ellos como personales, entonces yo ahí remito esa información al profesor..." (Entrevista 3). 
http://doi.org/10.15359/ree.24-3.15

http://www.una.ac.cr/educare

educare@una.ac.cr

Las fundamentaciones descritas no solo son destacadas por el profesorado, en función del beneficio directo que estas traen para sus estudiantes, sino que también son utilizadas para dar cuenta de la valoración que le atribuyen a su saber pedagógico que ha sido construido producto de los procesos de reflexión que han tenido como profesionales de la educación y a las innovaciones evaluativas, que difícilmente podrían haber realizado si no se desempeñaran en una escuela vulnerable.

Reconocen que desde el momento en que se han atrevido a innovar en su manera de evaluar, están mucho más alertas a observar si sus estudiantes están aprendiendo o no, a poner atención en las dificultades que tienen para aprender, a elaborar formatos evaluativos que faciliten su aprendizaje y utilicen el error para aprender, a conocer sus distintos estilos de aprendizaje, en fin, a ver la evaluación como una ayuda a los procesos de aprendizaje y no solo como una forma administrativa de evaluar el trabajo. Ello les permite resguardar posibles consecuencias emocionales desfavorables que se pueden presentar, como la frustración, el enojo y la baja disposición a aprender, características muy comunes en este contexto escolar.

No obstante, las condiciones descritas anteriormente, que vinculan las prácticas evaluativas del profesorado a la evaluación auténtica, también fundamentan este ámbito de la docencia desde la evaluación tradicional. Es decir, reconocen ciertas barreras que a veces resultan difíciles de sortear, especialmente aquellas vinculadas a los acotados tiempos de aula y la estricta administración escolar. En el primer caso, porque reconocen que este tipo de evaluación requiere emplear más tiempo que la tradicional. En el segundo, porque se sienten presionados por la normativa educacional y la administración escolar que les exige el cumplimento de un número determinado de calificaciones por semestre, a lo que se debe adicionar las exigencias propias de las pruebas institucionales y nacionales, lo que hace que, a veces, la preocupación por las notas se convierta en un tema central. Al menos así lo expresa una profesora: "... por eso yo trabajo con notas clase a clase y eso tiene un promedio, al final del mes o de la unidad y ahí se aplica la prueba institucional..." (Entrevista 4).

Sin embargo, se observa un consenso respecto de la importancia de ampliar este estrecho sentido de la evaluación tradicional, hacia la reflexión sobre la propia acción evaluativa y sobre el impacto (positivo o no) que tiene la evaluación en el estudiantado. El siguiente relato, da cuenta de lo expresado:

... resulta que hay niños que son más lentos y otros más rápidos, entonces algunos avanzaban de acuerdo a su ritmo, entonces a veces ocupábamos tres clases para terminar la evaluación, porque yo evaluaba la primera parte e iba pasando por mesa para ver lo que habían hecho hasta ese momento, después a la otra clase lo otro y finalmente a la tercera clase terminábamos con la nota. (Entrevista 8) 
http://doi.org/10.15359/ree.24-3.15

\section{b) Lecciones aprendidas del profesorado}

En esta categoría, se ha querido reunir un espacio de reflexión que emerge del discurso del profesorado que -más allá de lo que podría aparecer como una crítica a sus centros de formación docente- demuestra las dificultades que ha tenido que sortear para llegar a lo que hemos denominado una etapa en transición hacia la incorporación de prácticas evaluativas más auténticas en su quehacer profesional.

En esta categoría analítica, surgen cuatro subcategorías: Importancia de superar falencias en su formación inicial, necesidad de insertarse tempranamente en el sistema educativo, acercar la universidad al mundo escolar y valorar el rol del profesorado.

La importancia de superar falencias en su formación inicial aparece permanentemente en las reflexiones del profesorado, porque -a su juicio- constituye la fuente principal de sus concepciones profesionales tradicionales sobre la evaluación; con sus expresiones quieren no solo dar cuenta de las falencias en los cursos de evaluación contemplados en sus respectivas mallas curriculares, sino también, de las experiencias evaluativas que tuvieron que vivir como estudiantes de pregrado en las otras asignaturas cursadas, las que estaban estrechamente vinculadas a la evaluación tradicional. Tanto en las primeras como en las segundas, el profesorado destaca la distancia con concepciones evaluativas más holísticas y auténticas, así como su homogeneidad, monotonía y cercanía con las experiencias que han tenido que sortear en el mundo escolar. Esta situación es descrita por el profesorado como un verdadero abismo de dos realidades que no dialogan ni se intersectan en ninguna parte. Fundamentalmente, porque en la evaluación promovida en las instituciones de educación superior existe un gran ausente: los niños y niñas en formación y su realidad socioeducativa y sociocultural, como lo demuestra el siguiente relato:

Cuando uno llega a una escuela vulnerable, se da cuenta que existe un abismo entre lo estudiado en la universidad en los cursos de evaluación, lo vivido en las diferentes asignaturas de la malla curricular y la realidad que me tocó enfrentar en la escuela, porque como que los profesores en la universidad se olvidan que la evaluación está en función del aprendizaje de ellos y no se trata sólo de constatar si saben o no saben, sino ponerse en situación de cómo pueden aprender mejor y pensar en las mejores estrategias evaluativas para evaluar ese aprendizaje, que es lo importante. (Entrevista 6)

Esta es una de las razones por las cuales el profesorado efectúa recomendaciones a la formación inicial docente; abogan por una revisión de las mallas curriculares, una mayor preocupación por el análisis de los programas de estudio en donde reconocen que debería haber propuestas evaluativas muchos más pertinentes a las necesidades de sus estudiantes en consideración a los diferentes contextos escolares, como lo señala el siguiente relato: 
http://doi.org/10.15359/ree.24-3.15

http://www.una.ac.cr/educare

educare@una.ac.cr

Siento que debería fortalecerse la formación inicial, especialmente las mallas de formación que adolecen de muchas cosas desde el punto de vista evaluativo, de hecho, los programas del Ministerio de Educación de Chile han sido de ayuda para enfrentar los desafíos evaluativos. (Entrevista 3)

En este mismo contexto, el profesorado valora que a los futuros maestros y maestras se les ofrezca la posibilidad de insertarse tempranamente en el sistema educativo, tal como hoy lo exige la ley 20903 (Ministerio de Educación, 2016), que demanda, a las instituciones formadoras de docentes, la obligación de incorporar prácticas tempranas y progresivas en sus mallas de formación, generando convenios específicos para insertar a sus estudiantes anticipadamente en el mundo escolar. Lo que -a juicio del profesorado entrevistado- permitiría a los futuros cuerpos docentes tener una visión más adecuada de lo que efectivamente ocurre en la escuela y de las necesidades que posee el estudiantado. Concretamente el profesorado demanda:

Formar a los futuros profesores en un ambiente escolar real; observando clases apenas ingresan a las carreras de pedagogía e insertándolos tempranamente al contexto escolar, eso se debería fortalecer... yo siento que lo que debería fortalecerse es que ellos salieran más del aula porque entonces, en la práctica, la práctica va a fortalecer la formación que ellos puedan tener. (Entrevista 3)

Un grupo de docentes van más allá y propone que el futuro profesorado pueda observar las clases de personal más experimentado y, por sobre todo, que la universidad pueda tener un contacto más estrecho y colaborativo con el mundo escolar, que considere el saber pedagógico en evaluación del profesorado en ejercicio, y acerque la teoría y la práctica en evaluación y no solo desde el mundo didáctico como habitualmente se recomienda. Específicamente, el profesorado entrevistado aboga para que los docentes y las docentes en formación puedan presenciar las clases y las prácticas de los mejores profesores y profesoras de los establecimientos educacionales, para aprender de la forma en que enfrentan sus clases, los procedimientos didácticos y evaluativos utilizados y de cómo todo esto debe ser contextualizado al entorno escolar. Al respecto señalan:

Lo que nos pasa es porque las universidades no vienen a la escuela, los docentes de la universidad no vienen a la escuela, no hay ni un rector que venga a la escuela y diga "haber... hagamos esto". Las universidades no tienen estudios sobre lo que está pasando... ellos no hacen un trabajo con los liceos, no hacen trabajos con escuelas, para ver en terreno qué les está faltando a los profesores, ver que les falta a los estudiantes, etc., al menos eso pienso yo. (Entrevista 4)

Pero la necesidad de acercar la universidad a la escuela tiene -de acuerdo con el relato del profesorado- otra variable no menos importante, dado que han constatado que uno de los 
http://doi.org/10.15359/ree.24-3.15

factores que contribuye a esta falta de acercamiento tiene relación con la falta de experiencia en aula del profesorado universitario. Es decir, de las formadoras y los formadores de personal formador, quienes fundamentan sus clases en la teoría y no desde el mundo real ni desde la cotidianeidad de la escuela, la que han llegado a conocer en su trayectoria profesional:

En la universidad, los profesores no tenían experiencia de aula, entonces claro el discurso era más bien teórico y de ficción... y los alumnos [de pedagogía] nos dábamos cuenta, no era muy complejo darse cuenta de esa situación, porque aparte las teorías, la teoría que se aplicaba tampoco nos ayudaba mucho, insisto seguía siendo algo artificial, porque la realidad en la sala de clases es distinta. (Entrevista 5)

Las recomendaciones realizadas no hacen sino dar cuenta del complejo camino recorrido por el profesorado en sus años de ejercicio profesional, en el proceso de transición hacia la incorporación de estrategias evaluativas más auténticas, en donde han debido sortear las falencias de una formación inicial homogénea, la falta de consideración de la universidad con la escuela y la inexperiencia del profesorado universitario, desde el punto de vista evaluativo, de la realidad y complejidad que enfrentan en el aula. Variables que han sido capaces de aislar, entender y superar. De allí que al unísono terminan por valorar mucho más el rol del profesorado, que al final del relato reconocen como el verdadero artífice de un proceso que debió haber empezado tempranamente en sus procesos formativos. Pero que, como se ha demostrado, han tenido que aprender en su práctica con perseverancia y ahínco, en lo que llaman la compleja tarea de llegar a ser profesor o profesora. En las aulas reales los aspectos didácticos no están separados de los evaluativos ni del contexto escolar, ahí no basta con poner una calificación o evaluar mediante instrumentos descontextualizados y monótonos que buscan constatar la presencia o ausencia de un aprendizaje, la mayoría de las veces alejado del desarrollo de habilidades y actitudes para la vida, y en donde el profesorado debe ser un acompañante y no un juez en este proceso. Esto, porque en la tarea evaluativa no hay antagonismos sino un acompañamiento virtuoso en donde "uno va fiscalizando, monitoreando, aconsejando, acompañando al aprendiz en su propio contexto, tarea compleja que a la larga reivindica el rol del profesor..." (Entrevista 1).

\section{Discusión}

Al examinar los resultados, observamos que las personas participantes en el estudio se encuentran en un proceso de transición desde una práctica evaluativa tradicional a una cada vez más auténtica. Decimos en transición, porque han dado pasos significativos en este proceso, especialmente, al realizar una triangulación entre su formación inicial en evaluación, su experiencia profesional en el aula y los modelos pedagógicos que proponen implementar una evaluación más auténtica. 
http://doi.org/10.15359/ree.24-3.15

http://www.una.ac.cr/educare

educare@una.ac.cr

Aparece la convicción de que sus procesos formativos no han sido del todo satisfactorios, debido a que el modelo propuesto por la academia no considera las particularidades de los diferentes contextos escolares ni dialoga con el profesorado en ejercicio para conocer la realidad que les corresponde vivir en el aula. Coincidimos con Blanco Loro y González López (2010), al señalar que el profesorado se siente estimulado a buscar otros modelos evaluativos que les permitan enfrentar con mayor éxito su tarea, ya que tiene la convicción que la evaluación es una actividad ineludible, significativa y útil para el ejercicio de su actividad docente y para el aprendizaje de sus estudiantes.

En este importante proceso de reconstrucción, el profesorado concuerda con lo expresado por Vallejo Ruiz y Molina Saorín (2014), en dos cuestiones fundamentales: la primera, en la necesidad de contrastar sus propios procesos formativos con su experiencia de aula y, la segunda, en vincular sus praxis evaluativas con asuntos relevantes de la vida de sus jóvenes estudiantes, promoviendo una evaluación más afín con sus características y necesidades y pertinente al contexto escolar vulnerable donde se insertan.

El profesorado es consciente de que este proceso conlleva un importante cambio, el cual ha implicado que su propio saber pedagógico se haya ido modificando, especialmente en función de lo que destaca Moreno Olivos (2017), esto es, atendiendo a las características, habilidades y necesidades de sus estudiantes y a su contexto escolar.

Observamos que dentro de los cambios experimentados emerge, con fuerza, la sensación de una mayor valoración de aquellas prácticas evaluativas que les permiten tomar más y mejores decisiones, y que consideran el trabajo colaborativo, la autoafirmación personal y el desarrollo de habilidades para la vida de sus estudiantes, mediante instrumentos que validan tanto la autoevaluación, como la coevaluación y la heteroevaluación, además de aportar información relevante y pertinente para la reflexión y toma de decisión de docentes y estudiantes.

En cuanto a la consideración del estudiantado en los procesos evaluativos, es destacable que el profesorado -tal y como señalan estudios previos (Montes Ayala y Torres González, 2017; Rodríguez Moneo y Huertas, 2017)- destaca la importancia de incluir algunos recursos afectivos como herramienta mediadora de la confianza y diálogo entre docentes y estudiantes, y estudiantes y sus pares, a quienes se esfuerzan por dar un trato más humano. Son especialmente significativas sus reflexiones respecto a la importancia que poseen estos recursos afectivos en la disposición del estudiantado para aprender y para la evaluación de sus aprendizajes. También resulta interesante, la valoración que hace el profesorado de la importancia que posee, en los procesos evaluativos, el compromiso estudiantil con sus tareas de aprendizaje; al respecto se coincide con Arguello Torres et al. (2017), en que las escuelas también necesitan desarrollar en sus estudiantes tanto sus capacidades como sus sentimientos y convicciones. 
http://doi.org/10.15359/ree.24-3.15

A pesar de que la bibliografía reconoce que la evaluación auténtica supone la modificación de las prácticas evaluativas del profesorado (Díaz Barriga y Barroso Bravo, 2014), en el caso del grupo estudiado, esto no siempre es así. Principalmente porque, aunque son capaces de reflexionar respecto a la importancia y bondades que ofrece este modelo evaluativo auténtico, no siempre se llega a implementar un ciclo evaluativo como lo recomendado por Brown (2015) o Vallejo Ruiz y Molina Saorín (2014), por dos razones: las barreras contextuales y el uso de los resultados.

En primer lugar, se reconoce que existen ciertas barreras contextuales que no siempre son favorables al trabajo evaluativo, especialmente resulta crítica la escasez de tiempo y la necesidad de satisfacer un número determinado de calificaciones por semestre, que lleva a diseñar instrumentos que, en su mayoría, incorporan preguntas de fácil corrección (por ejemplo, selección múltiple, términos pareados y verdadero/falso). En segundo lugar, a pesar de que declaran que es importante tomar decisiones a partir de los resultados obtenidos, tal como lo recomienda la bibliografía (Santos Guerra, 2015), esto no siempre lo realizan, lo que hace que el profesorado pierda una valiosa oportunidad de retroalimentar a sus estudiantes y conectar, como recomiendan López-Lozano et al. (2017) “los resultados de la evaluación con los procesos de aprendizaje y las decisiones que se toman sobre la planificación de la enseñanza" (p. 862), o como diría Díaz Barriga y Barroso Bravo (2014), con la educación para la vida.

La incapacidad para concluir a cabalidad un ciclo evaluativo hace que el profesorado transite entre un rol tradicional y un rol de mediador y facilitador de diálogos, reflexiones y acuerdos conjuntos entre estudiantes, como efectivamente debería ser (Rittaco Real y Amores Fernández, 2016). Fenómeno que ha sido estudiado y calificado como complejo, puesto que exige múltiples, constantes y agotadoras transiciones del profesorado, en la medida en que supera los distintos obstáculos de aprendizaje (López-Lozano et al., 2017), los mismos que el profesorado ha tenido que enfrentar a lo largo de su trayectoria profesional y de cuya carencia responsabiliza a la formación inicial, más allá del manejo del contenido disciplinar y de su didáctica (Ibáñez Salgado, 2014).

\section{Conclusiones}

El saber pedagógico evaluativo del profesorado que se desempeña en escuelas vulnerables de la comuna de Arica se encuentra en un proceso de transición desde el modelo tradicional de evaluar hacia la propuesta de evaluación auténtica, y evidencia que las prácticas evaluativas tradicionales aún siguen presentes en la acción docente.

De ahí que los resultados demuestren una tensión entre el saber pedagógico en evaluación y las prácticas docentes en ese ámbito. Esto, debido a que, aunque el profesorado entrevistado declara realizar sus prácticas evaluativas desde la evaluación auténtica, al profundizar en el análisis, se advierte que no son pocas las ocasiones en que en sus prácticas de aula terminan evaluando desde el modelo tradicional. Es decir, más allá de la creencia construida y expresada 
http://doi.org/10.15359/ree.24-3.15

http://www.una.ac.cr/educare

educare@una.ac.cr

por el profesorado en el contexto de vulnerabilidad estudiado, la evaluación escolar sigue siendo un mecanismo de control que busca encasillar las acciones del alumnado, clasificándolo en bueno y malo a partir de estándares predeterminados (Vaillant, 2008).

De hecho, si bien señalan que han incorporado evaluaciones formativas, autoevaluaciones y ahora evalúan de manera más práctica, al profundizar en sus relatos se advierte que principalmente califican al estudiantado para responder a las exigencias evaluativas del establecimiento o medio escolar, y valoran, así, como un "logro" profesional el registro de notas.

Los resultados de la investigación relevan la experiencia docente en el proceso de construcción del saber pedagógico evaluativo, pero, sobre todo, la capacidad del profesorado para sintonizar con las necesidades de sus estudiantes y tener una lectura correcta en la consideración del medio socioeducativo en que se desempeñan, lo cual es especialmente significativo si se trata de un contexto vulnerable. Todo ello le permitiría una evaluación más holística, con un sentido integrador, pertinente y centrada en quienes aprenden.

Resulta interesante consignar que, si bien el profesorado en estudio releva como variables clave en el proceso de construcción de su saber pedagógico en evaluación, su formación inicial y su experiencia profesional, también declara la necesidad de vincular este proceso, tal como lo señala Pereira-Chaves (2015), con los requerimientos de sus estudiantes. Esto es, atendiendo especialmente a las particularidades del contexto escolar vulnerable y considerando otras prácticas evaluativas reales del profesorado. Es importante destacar que, si bien el profesorado manifiesta que, a través del tiempo, sus prácticas evaluativas se han transformado en una acción pedagógica que favorece el diálogo entre el profesorado y estudiantes, entre estudiantes entre sí y entre estudiantes y sus padres, madres o personas apoderadas, en el análisis de sus relatos se advierte que existen otros factores que inciden en el quehacer evaluativo del profesorado en el aula. Por ejemplo, un factor que obstaculiza esta transformación es la falta de tiempo escolar, que no les permita completar a cabalidad un ciclo evaluativo, les hace perder una valiosa oportunidad de retroalimentar a sus estudiantes y mejorar su aprendizaje.

Esta evidente tensión del profesorado desafía el rol del personal directivo de los establecimientos escolares, y le invita a analizar y visibilizar las prácticas evaluativas del profesorado y, principalmente, acompañarle en el ajuste entre el saber pedagógico en evaluación y lo que realmente realiza en el aula. Seguramente será parte de este reto reconsiderar el número de calificaciones que se les exige registrar durante el semestre, así como la posibilidad de redistribuir algunas tareas cotidianas, con el fin de permitir a los cuerpos docentes disponer de mayor tiempo para la retroalimentación de sus estudiantes.

No obstante, este estudio tiene algunas limitaciones que sugieren que los resultados deben interpretarse con cautela y como una primera aproximación al campo. Un abordaje más integral de los centros educativos vulnerables, así como de las prácticas pedagógicas y, 
http://doi.org/10.15359/ree.24-3.15

en específico, evaluativas del profesorado debería, necesariamente, incorporar a otros actores claves de la comunidad educativa (por ejemplo, alumnado, personal directivo, agentes políticos, familias). Asimismo, un enfoque cuantitativo basado en un gran estudio de encuestas de población nos permitiría verificar y validar los resultados que hemos obtenido, así como desarrollar modelos predictivos para apoyar la toma de decisiones sobre políticas educativas encaminadas a la mejora de la formación del profesorado, así como a la reducción del fracaso escolar y la exclusión educativa.

En cualquier caso, los resultados obtenidos resultan realmente pertinentes para el sistema educativo chileno, inmerso, como comentábamos al inicio, en la búsqueda constante de elementos y mecanismos que contribuyan a mejorar la calidad de la educación.

Vincular los procesos de formación inicial docente con las prácticas evaluativas reales del profesorado que se desempeña en diversos contextos escolares constituye un desafío que políticos, prácticos y teóricos deben abordar. Se requiere conocer y relevar el saber pedagógico del profesorado y, con ello, nutrir los itinerarios formativos de las carreras de pedagogía y sus respectivos programas de estudios, con el fin de rejuvenecer los procesos de formación docente desde una perspectiva que considera y valora las prácticas educativas realizadas en multiplicidad de contextos educativos. Saben que hay que hacerlo, tienen las herramientas para hacerlo, pero en no pocas ocasiones se quedan más bien en el intento.

\section{Referencias}

Arguello Torres, L. V., Filian Hurtado, F. H., Guevara Espinoza, J. C. y Cadena Hurtado, L. A. (2017). La importancia de la determinación de indicadores de desarrollo. La unidad de lo afectivo y lo cognitivo. Revista Didasc@ lia: Didáctica y Educación, 8(1), 193-206. https://dialnet. unirioja.es/servlet/articulo?codigo $=6630554$

Blanco Loro, M. E. y González López, I. (2010). Políticas evaluativas del profesorado de primaria, condicionantes de su actividad docente. Bordón, 62(1), 29-47. https://dialnet.unirioja.es/ descarga/articulo/3178524.pdf

Brown, R. (2015). La evaluación auténtica: El uso de la evaluación para ayudar a los estudiantes a aprender. RELIEVE-Revista Electrónica de Investigación y Evaluación Educativa, 21(2), 1-10. https://doi.org/10.7203/relieve.21.2.7674

Cárdenas Pérez, A. V., Soto Bustamante, A. M., Dobbs-Díaz, E. y Bobadilla-Goldschmidt, M. (2012). El saber pedagógico: Componentes para una reconceptualización. Educación y Educadores, 15(3), 479-496. https://www.scielo.org.co/scielo.php?script=sci arttext\&pid $=$ S0123-12942012000300008 
http://doi.org/10.15359/ree.24-3.15

http://www.una.ac.cr/educare

educare@una.ac.cr

Carrero, V., Soriano, R. M., y Trinidad, A. (2012). Teoría fundamentada "Grounded Theory" (Colección

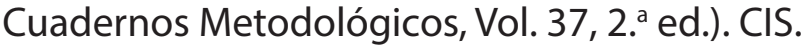

Díaz Barriga, F. y Barroso Bravo, R. (2014). Diseño y validación de una propuesta de evaluación auténtica de competencias en un programa de formación de docentes de educación básica en México. Perspectiva Educacional, 53(1), 36-56. https://www.perspectivaeducacional.cl/ index.php/peducacional/article/view/210

Díaz López, C. y Pinto Loría, M. de L. (2017). Vulnerabilidad educativa: Un estudio desde el paradigma socio crítico. Praxis Educativa, 21(1), 46-54. http://dx.doi.org/10.19137/ praxiseducativa-2017-210105

Forján, R. y Morelatto, G. (2018). Estudio comparativo de factores de resiliencia en docentes de contextos socialmente vulnerables. Psicogente, 21(40), 277-296. https://doi.org/10.17081/ psico.21.40.3075

Hernández-Nodarse, M. (2017). ¿Por qué ha costado tanto transformar las prácticas evaluativas del aprendizaje en el contexto educativo? Ensayo crítico sobre una patología pedagógica pendiente de tratamiento. RevistaElectrónica Educare, 21(1), 1-27.https://doi.org/10.15359/ ree.21-1.21

Herrera, J. (2017). La investigación cualitativa. http://biblioteca.udgvirtual.udg.mx:8080/jspui/ bitstream/123456789/1167/1/La\%20investigaci\%c3\%b3n\%20cualitativa.pdf

Ibáñez Salgado, N. (2014). Saberes profesionales para la transformación del modelo educativo en Chile. Estudios pedagógicos, 40(Especial), 145-160. https://doi.org/10.4067/S0718$\underline{07052014000200009}$

Infante, P., Rujano, R. y Sáez, R. (2011). Teoría fundamentada: Una visión crítica. Revista Trabajo Social, 13, 33-53. https://revistas.udea.edu.co/index.php/revistraso/article/ view/24187/19764

López-Lozano, L., Solís, E. y Fernández-Arroyo, J. (2017). Los cambios en las concepciones sobre evaluación en ciencias de futuros maestros de primaria. Revista Complutense de Educación, 29(3), 847-864. https://doi.org/10.5209/RCED.54061

Ministerio de Educación. (01 de abril de 2016). Ley 20903. Crea el sistema de desarrollo profesional docente y modifica otras normas. Biblioteca del Congreso Nacional de Chile, pp. 1-62. https://www.leychile.cl/Navegar?idNorma $=1087343$

Monge Acuña, V. (2015). La codificación en el método de investigación de la Grounded Theory o teoría fundamentada. Innovaciones educativas, 17(22), 77-84. https://doi.org/10.22458/ ie.v17i22.1100 
http://doi.org/10.15359/ree.24-3.15

http://www.una.ac.cr/educare educare@una.ac.cr

Montes Ayala, M. y Torres González, J. A. (2017). Las competencias socio - afectivas docentes y la formación para la práctica educativa del desarrollo personal y para la convivencia, en el marco de la educación inclusiva. Revista de Educación Inclusiva, 8(3), 271-284. http:// revistaeducacioninclusiva.es/index.php/REl/article/view/101

Moreno Olivos, T. (2017). La evaluación, ¿nos conducirá a la tierra prometida? Perspectiva Educacional, 56(1), 147-163. $\quad$ http://www.perspectivaeducacional.cl/index.php/ peducacional/issue/view/36

Murillo, F. J. e Hidalgo, N. (2016). Evaluación democrática y para la democracia. Revista Iberoamericana de Evaluación Educativa, 9(1), 5-7. https://dialnet.unirioja.es/descarga/ articulo/5534513.pdf

Noreña, A. L., Alcalaz-Moreno, N., Rojas, J. G., y Rebolledo-Malpica, D. (2012). Aplicabilidad de los criterios de rigor y éticos en la investigación cualitativa. Aquichan, 12(3), 263-274. http:// jbposgrado.org/icuali/Criterios\%20de\%20rigor\%20en\%20la\%20Inv\%20cualitativa.pdf

Perassi, Z. (2014). Las prácticas evaluativas de docentes en ejercicio. Escuela "innovadora" vs. escuela "tradicional". Alteridad. Revista de Educación, 9(1), 44-55. https://doi.org/10.17163/ alt.v9n1.2014.04

Pereira-Chaves, J. (2015). Evaluación, medición o verificación de los aprendizajes en el aula: Un estudio de caso en el Colegio Humanístico Costarricense de Heredia. Revista Electrónica Educare, 19(2), 405-428. https://doi.org/10.15359/ree.19-2.22

Quesada Serra, V., Rodríguez Gómez, G. e Ibarra Sáiz, M. S. (2017). Planificación e innovación de la evaluación en educación superior: La perspectiva del profesorado. Revista de Investigación Educativa, 35(1), 53-70. https://doi.org/10.6018/rie.35.1.239261

Ravela, P. (2015). Consignas, devoluciones y calificaciones: Los problemas de la evaluación en las aulas de educación primaria en América Latina. Páginas de Educación, 2(1), 49-89. https:// doi.org/10.22235/pe.v2i1.703

Reyes, L., Campos, J., Osandón L. y Muñoz, C. (2013). El profesorado y su rol en la formación de los nuevos ciudadanos: Desfases entre las comprensiones, las actuaciones y las expectativas. Estudios Pedagógicos, 39(1), 217-237. https://doi.org/10.4067/S0718-07052013000100013

Rittaco Real, M. y Amores Fernández, F. J. (2016). Percepciones del profesorado y alumnado del programa de cualificación profesional inicial (PCPI). Desarrollo del proceso de enseñanzaaprendizaje en programas de prevención del fracaso escolar en secundaria. Espiral. Cuadernos del profesorado, 9(18), 3-15. https://doi.org/10.25115/ecp.v9i18.1000 
http://doi.org/10.15359/ree.24-3.15

http://www.una.ac.cr/educare

educare@una.ac.cr

Rodríguez Moneo, M. y Huertas, J. A. (2017). Motivación y cambio conceptual. Tarbiya, Revista de Investigación e Innovación Educativa, 26, 51-71. https://revistas.uam.es/tarbiya/article/ view/7115

Sabino, C. A. (2014). El proceso de investigación. Editorial Episteme.

Santelices, M. V., Galleguillos, P., González, J. y Taut, S. (2015). Un estudio sobre la calidad docente en chile: El rol del contexto en donde enseña el profesor y medidas de valor agregado. Psykhe (Santiago), 24 (1), 1-14. http://dx.doi.org/10.7764/psykhe.23.2.673

Santos Guerra, M. Á. (2015). Corazones, no solo cabezas en la universidad. Los sentimientos de los estudiantes ante la evaluación. REDU. Revista de Docencia Universitaria, 13(2), 125-142. https://doi.org/10.4995/redu.2015.5441

Solar, M. I. y Díaz, C. (2018). El profesor universitario: Construcción de su saber pedagógico e identidad profesional a partir de sus cogniciones y creencias. Calidad en la Educación, 30, 208-232. https://doi.org/10.31619/caledu.n30.178

Sotos, M. y López, M. C. (2015). El proceso de construcción del saber pedagógico en educación matemática: El caso de María Antònia Canals. Épsilon, 90, 59-69. http://thales.cica.es/epsilon/ sites/thales.cica.es.epsilon/files/\%5Bfield volumen-formatted\%5D/epsilon90 6.pdf

Strauss, A. y Corbin, J. (2002). Bases de la investigación cualitativa. Técnicas y procedimientos para desarrollar la teoría fundamentada (E Zimmerman, Trad.). Editorial Universidad de Antioquia.

Suárez, D. H. (2017). Docentes, relatos de experiencia y saberes pedagógicos. La documentación narrativa de experiencias en la escuela. Investigación Cualitativa, 2(1), 42-54. http://dx.doi. org/10.23935/2016/01034

Tardif, M. (2004). Los saberes del docente y su desarrollo profesional (Vol. 97). Narcea.

Torres, R. (2018). Tackling inequality? Teacher effects and the socioeconomic gap in educational achievement. Evidence from Chile. School Effectiveness and School Improvement, 29(3), 383-417. https://doi.org/10.1080/09243453.2018.1443143

Vaillant, D. (2008). Algunos marcos referenciales para la evaluación del desempeño docente en América Latina. Revista Iberoamericana de Evaluación Educativa, 1(2), 7-22. https://revistas. uam.es/index.php/riee/article/view/4663/5100

Vallejo Ruiz, M. y Molina Saorín, J. (2014). La evaluación auténtica de los procesos educativos. Revista Iberoamericana de Educación Educativa, 64, 11-25. https://doi.org/10.35362/ $\underline{\text { rie640403 }}$ 